\title{
Modeling study on the combustion of intumescent fire-retardant polypropylene
}

\author{
F. Zhang, J. Zhang*, Y. Wang \\ Key Laboratory of Rubber-Plastics for Ministry of Education, Qingdao University of Science and Technology, 53 Zheng \\ Zhou Road, Qingdao, 266042, P.R. China
}

Received 13 February 2007; accepted in revised form 5 March 2007

\begin{abstract}
The heat transfer and burning behavior of intumescent fire-retardant polypropylene were studied by cone calorimeter at heat flux levels of $50 \mathrm{~kW} \cdot \mathrm{m}^{-2}$ to establish an essential physical model for the intumescence process in fire. A mathematical model for the burning process of fire-retardant intumescent polymer was put forward based on the assumption that an intumescent front existed between the char layer and virgin layer. The model emphasizes the thermodynamic aspect of the intumescence process and a corresponding submodel is presented. Meanwhile the thicknesses and mass loss rates of the intumescent polypropylene during burning were measured for the validation of the modeling results. Thermal conductivity and heat capacity of polymer material were also measured as input parameters of the model. The validation results showed that the intumescent thicknesses and mass loss rates predicted by the model were in good agreement with the experimental results. The model was also used to predict the temperature distribution across the sample thickness during burning. The study shows that the present model can appropriately describe the intumescent behavior of the polymer and numerically predict its mass loss rates and temperature distribution in fire.
\end{abstract}

Keywords: modeling and simulation, intumescence, polymer burning, cone calorimeter, polypropylene

\section{Introduction}

Polypropylene (PP) is widely used in many fields such as housing materials, transport or electrical engineering applications. The polymer is inherently flammable due to its chemical constitution and flame retardancy is usually required in its applications. Halogen containing fire retardants were widely used for PP due to their high efficiency. However, the toxicity and corrosiveness of the combustion products and smoke generated from these fire reatradants have limited their further development. As an alternative way to improve the polymer's fire resistance, the intumescent fire retardant systems are growing rapidly[1-4].

Intumescent fire retardant materials are halogenfree, and provide flame retardant properties by developing a carbonaceous shield (char) on the sur- face protecting the underlying material from the action of the incident heat flux. The resulting char reduces the heat transfer to the substrate. Generally, intumescent formulations include three major components [5, 6]: 1 - acid source, such as phosphate; 2 - carbonific agent such as polyols; 3 - blowing agent such as melamine. When exposed to the heat flux from a fire, the temperature within these materials rises, causing the thermoplastic to melt. At a critical temperature, an endothermal chemical reaction releases volatiles, which results in swelling of the intumescent layer to many times of its original thickness. There are several reactions occurring almost simultaneously, but in a proper sequence during swelling. First, the acid source must decompose to yield an inorganic acid. Then it takes part in the dehydration of the carbon source to yield the

*Corresponding author, e-mail: safetyqust@163.com

(c) BME-PT and GTE 
carbon char. Finally, the blowing agent decomposes to yield gaseous products that cause the char to swell and hence provide the thermal barriers to protect the underlying materials.

The processes taking place during intumescence were studied mainly from the chemical viewpoint. However, in recent years the number of reliable numerical models developed to predict the burning behavior of intumescent fire retardant materials is growing. Proper modeling can provide a useful means to simulate the influence of various parameters on the efficiency of intumescence and help to develop intumescent fire retardant.

Several models [5, 7-11] have been developed to study the effects of intumescence on heat transfer to the underlying surface. Most models are onedimensional, and concentrate on the effects of swelling on the thermal properties of the materials. The one-dimensional models have been developed to investigate intumescent behavior with energy and mass conservations. The thickness of virgin material and char layers and the location of the pyrolysis zone are functions of the time, and each layer is assigned its own values of thermodynamic parameters. All of these models rely on empirical information such as the size and rate of expansion. For these models, it is generally believed that two mechanisms are responsible for slowing the transport of heat. One is that the pyrolysis reaction is endothermal, causing energy to be absorbed during swelling phase. Another one is by reducing thermal conductivity of the intumescent char. Staggs [12] has described a model of volatile transport and has discussed the effects of large changes in volume. Several studies [13] of solid phase decomposition and char forming are indicative of the complex processes.

Two or three-dimensional intumescent models have also been developed. Bhargava et al. [14] studied the heat transfer in two dimensions through epoxy based fire resistant coatings. In their model, only energy equations were incorporated while the endothermal effect was ignored. A three-dimensional approach was investigated at NIST [15], in which bubbling, melt hydrodynamics, heat transfer and chemical reactions were incorporated. The bubbles obey equations of mass, momentum, and energy on an individual basis according to the values of local parameters, and their collective behavior is responsible for the swelling and fire retardant properties of the material. The number of bubbles must be added as a known parameter value, but the fact is that it is hard to know the number. It is only an empirical value. All these shortcomings limit the model's further development.

In this paper, the heat transfer and burning behaviors of the intumescent fire-retardant polypropylene were studied using cone calorimeter, DSC, and DRX-I thermal conductivity tester to establish an essential physical model for the intumescence process in fire. A mathematical model for the burning process of fire-retardant intumescent polymer was put forward based on the assumption that an intumescent front existed between the char layer and virgin layer. In particular, an intumescent heat absorption model was incorporated to include thermodynamic swelling work involved in the intumecent process. Several important parameters were measured as input values of the model.

\section{Modeling development}

\subsection{Physical Model Description}

When an intumescent fire-retardant material is exposed to an external heat source, the initial heat transfer mode on the material is dominated by heat conduction and this is a preheating stage. The temperature of the material increases continuously with time under the influence of the external heat flux. When the temperature of the material reaches the temperature of intumescence, a thin intumescent layer is formed on the material surface. The intumescent reaction will occur within the intumescent layer. The incombustible and combustible gases will release from the intumescence layer, which make the viscoelastic fluid expand. Intumescent interface gradually moves into the inside of the material, with residual char left. This stage is the intumescent burning stage. So the material can be divided into three independent layers, i.e. char layer, intumescence layer and virgin layer, as shown in Figure 1. On heating, the intumescence

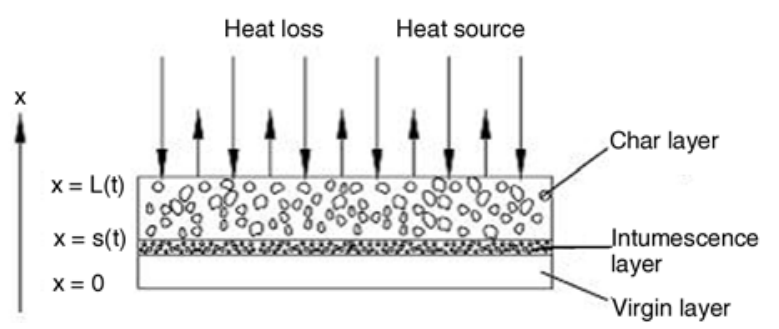

Figure 1. Schematic diagram of one-dimensional intumescent polymer model 
develops and the intumescent front interface moves into the material from the top $(x=L(t))$ to the bottom $(x=0)$. The position of the front is defined by its location at $x=s(t)$. As the intumescent front interface moves through the material, the change of volume as char is formed will push the boundary of the top surface $x=L(t)$ upwards.

\subsection{Intumescent heat absorption submodel}

One of the intumescent fire-retardant mechanisms is heat absorption within intumescent layer. The heat absorption may primarily come from two sources, i.e. pyrolysis heat of the material including phase change heat and intumescent heat absorption. The latter is a peculiar phenomenon occurring during intumescence and has rarely been investigated so far. In this study, an intumescent heat absorption submodel was developed and coupled to describe intumescence mechanism.

The submodel is based on the following simplifying assumptions: 1 - the volatile gases behave ideally; 2 - intumescence reaction occurs at a definite temperature with infinitely thin layer; 3 - there is a thermodynamic equilibrium state between the intumescence layer and surroundings. So the infinitely thin intumescence layer can be approximately treated as an isothermal reversible system. Figure 2 shows intumescent volume change from state 1 ( $n$, $\left.P_{1}, V_{1}, T\right)$ to state $2\left(n, P_{2}, V_{2}, T\right)$.

It is well known that when ideal gas expands from initial state $1\left(n, P_{1}, V_{1}, T\right)$ to state $2\left(n, P_{2}, V_{2}, T\right)$ in an isothermal reversible way, the work in the process is reversible work, and

$W=-\int_{V_{g_{1}}}^{V_{g_{2}}} p \mathrm{~d} V$

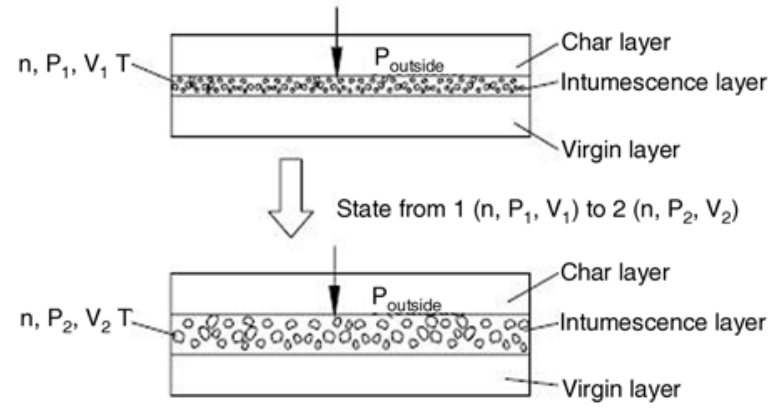

Figure 2. Intumescent volume change from state 1 ( $n, P_{1}$, $\left.V_{1}, T\right)$ to state $2\left(n, P_{2}, V_{2}, T\right)$ where $p$ represents internal pressure. Replace ideal gas state equation $p v=n R T$ with the Equation (1), the Equation (2) is deduced:

$$
W=-\int_{V_{g_{1}}}^{V_{g_{2}}} \frac{n R T}{V} \mathrm{~d} V=n R T \ln \left(\frac{V_{g_{1}}}{V_{g_{2}}}\right)=\frac{m_{g}}{M_{g}} R T \ln \left(\frac{V_{g_{1}}}{V_{g_{2}}}\right)
$$

According to the first law of thermodynamics $\Delta U=Q+W$, with $\Delta U=0$ for ideal gas isothermal process. So the heat absorption, $Q$, in the isothermal reversible process is (3):

$$
Q=-W=-\frac{m_{g}}{M_{g}} R T \ln \left(\frac{V_{g_{1}}}{V_{g_{2}}}\right)=\frac{m_{g}}{M_{g}} R T \ln \left(\frac{V_{g_{2}}}{V_{g_{1}}}\right)
$$

Suppose that intumescence semi-fluid solid's volume is $V$, the porosity is $\phi$, then the gas volume is $V \cdot \phi$, accordingly (4):

$Q=\frac{m_{g}}{M_{g}} R T \ln \left(\frac{\phi_{2} V_{2}}{\phi_{1} V_{1}}\right)$

When the sample is flamed out, we suppose that the residual char yield will not change with the depth of the sample. So the residual char yield, char\%, is the same at the same experimental conditions (5):

$m_{g}=m_{0}(1-\operatorname{char} \%)$

Substituting Equation(5) in Equation (4), we obtain:

$$
Q=\frac{m_{0}(1-\mathrm{char} \%)}{M_{g}} R T \ln \left(\frac{\phi_{2} V_{2}}{\phi_{1} V_{2}}\right)
$$

For simplification, we suppose porosity $\phi$ will not change during intumescence. So Equation (6) can be simplified as Equation (7).

$$
Q=\frac{m_{0}(1-\operatorname{char} \%)}{M_{g}} R T \ln \left(\frac{V_{2}}{V_{1}}\right)
$$

As per unit mass semi-fluid solid expands from $V_{1}$ to $V_{2}$, the heat absorption needed, $Q$, is (8): 


$$
\begin{aligned}
Q= & \frac{1}{m_{0}}\left[\frac{m_{0}(1-\operatorname{char} \%)}{M_{g}} R T \ln \left(\frac{V_{2}}{V_{1}}\right)\right]= \\
& \frac{1-\operatorname{char} \%}{M_{g}} R T \ln \left(\frac{V_{2}}{V_{1}}\right)
\end{aligned}
$$

As per unit mass semi-fluid solid expands one unit volume, the heat absorption needed, $q_{i}$, is (9):

$q_{i}=\frac{\ln 2}{M_{g}} R T(1-\operatorname{char} \%)$

As reported previously [11], it is assumed that the generated gas composition is approximated by a mixture of $50 \%$ (wt) $\mathrm{CO}_{2}$ and $50 \%(w t) \mathrm{H}_{2} \mathrm{O}$. The generated gas molar mass $M_{g}$ can be expressed by the Equation (10):

$M_{g}=50 \% \cdot M_{\mathrm{H}_{2} \mathrm{O}}+50 \% \cdot M_{\mathrm{CO}_{2}}$

Therefore, the generated gas molar mass $M_{g}$ was taken as equal to $31 \mathrm{~g} \cdot \mathrm{mol}^{-1}$.

The pyrolysis temperature of PP occurs at about 553 623 K [16]. If we take the intumescence temperature as $600 \mathrm{~K}, \quad \operatorname{char} \%=31.94 \%, \quad M_{g}=$ $31 \mathrm{~g} \cdot \mathrm{mol}^{-1}$, and input these parameter values into Equation (9), the result of $q_{i}$ is $7.59 \cdot 10^{4} \mathrm{~J} \cdot \mathrm{kg}^{-1}$. In literature $[11,17]$ the values of pyrolysis heat reported for $\mathrm{PP}$ are variable, ranging from $50 \mathrm{~J} \cdot \mathrm{kg}^{-1}$ to $1.3 \cdot 10^{6} \mathrm{~J} \cdot \mathrm{kg}^{-1}$. These differences in pyrolysis heat may be caused by different experiment conditions, however, it is clear that more accurate pyrolysis heat of PP is needed. The calculation results in Equation (9) show that the intumescent heat absorption for PP is of importance comparing with that of pure PP.

\subsection{Intumescent velocity submodel}

For intumescent velocity $u$, it requires an equation to relate the velocity to other parameters. Basically, a momentum balance would be performed to obtain the relationship between the pressure generated by the volatile products, the bulk viscosity of the semifluid solid, and the velocity. However, the pressure field and viscosity are not easily attainable experimentally [15]. In this paper, we adopted an alternative approach to determine intumescent velocity by relating it to moving velocity $\partial s / \partial t$ of intumescent front interface. Under certain experimental conditions, intumescent velocity $u$ is a function of intu- mescent front interface movement velocity. We define an intumescent ratio $\Delta v_{f} / v_{0}$, where $\Delta v_{f}$ is the difference between the initial volume $v_{0}$ and the final volume $v_{f}, v_{0}$ is the initial volume of sample, $d$ is the initial depth of sample, and $D$ is the final depth of sample.

Assuming that the intumescent direction of material is one-dimensional (11), (12):

$\frac{D}{d}=\frac{v_{f}}{v_{0}}$

$\frac{D-d}{d}=\frac{v_{f}-v_{0}}{v_{0}}=\frac{\Delta v_{f}}{v_{0}}$

Equation (12) can be changed into Equation (13):

$D-d=\frac{\Delta v_{f}}{v_{0}} d$

differentiating Equation (13) with respect to time (14):

$\frac{\partial(D-d)}{\partial t}=\frac{\Delta v_{f}}{v_{0}} \frac{\partial d}{\partial t}$

Where the left side of Equation (14) is $u$, and $\partial d / \partial t$ equals to $\partial s / \partial t$. So Equation (14) can be changed into Equation (15):

$u=-\frac{\Delta v_{f}}{v_{0}} \frac{\partial s}{\partial t}$

Thus, the intumescent heat absorption submodel and intumescent velocity submodel can be incorporated into the following intumescent mathematical model.

\subsection{Intumescent mathematical model}

Intumescent combustion process is very complicated and the proposed model can not include all of the factors that influence the process. The present mathematical model is based on the conservation of energy with the following simplifiying assumptions: 1 - the model is one-dimensional; 2 - there is no heat exchange between generated gas and char; 3 - the thermal properties such as density, heat capacity, thermal conductivity are independently constant at char layer and virgin layer; 4 - the temperature at the interface of char layer and virgin layer $x=s(t)$ is the intumescence (pyrolysis) temperature; 5 - moisture content is ignored; 6 - intumescent layer is very thin, and turns into char layer 
quickly, and heat absorption includes pyrolysis heat and intumescent heat absorption.

Preheating stage:

The initial heat transfer mode on the material is dominated by heat conduction (16).

$\frac{\partial T}{\partial t}=\frac{k_{v}}{\rho_{v} c_{v}} \cdot \frac{\partial^{2} T}{\partial x^{2}}$

Boundary conditions (17), (18):

$x=0, k_{v} \frac{\partial T}{\partial x}=0$

$x=L, k_{v} \frac{\partial T_{v}}{\partial x}=a_{v} \dot{q}^{\prime \prime}-h_{v}\left(T_{s}-T_{\infty}\right)-\varepsilon_{v} \sigma\left(T_{s}^{4}-T_{\infty}^{4}\right)$

Initial condition (19):

$t=0, T=T_{\infty}$

\section{Intumescent combustion stage:}

When the top surface of the sample reaches intumescence (pyrolysis) temperature, intumescence stage starts. The material is divided into three layers: char layer, intumescent layer, virgin layer.

1) Char layer

Energy conservation (20):

$\frac{\partial T}{\partial t}+u \frac{\partial T}{\partial x}=\frac{k_{c}}{\rho_{c} c_{c}} \cdot \frac{\partial^{2} T}{\partial x^{2}}$

Boundary conditions (21), (22):

$$
\begin{aligned}
x=L(t), & k_{c} \frac{\partial T}{\partial x}= \\
& a_{c} \dot{q}^{\prime \prime}-h_{c}\left(T_{s}-T_{\infty}\right)-\varepsilon_{c} \sigma\left(T_{s}^{4}-T_{\infty}^{4}\right)
\end{aligned}
$$

$x=s(t), T=T_{p}$

$T_{S}$ represents the temperature of char layer surface, $s(t)$ represents the location of intumescent layer.

2) Intumescent layer $(x=s(t))$

Energy conservation (22), (23):

$k_{c} \frac{\partial T_{c}}{\partial t}=k_{v} \frac{\partial T_{v}}{\partial t}-\rho_{v} q_{p} \frac{\partial s}{\partial t}+u \rho_{v} q_{i}$

Intumescent velocity is equal to Equation (15).

Mass loss rate (24): $\dot{m}^{\prime \prime}=-\left(\rho_{v}-\rho_{c}\right) \cdot \frac{\partial s}{\partial t}$

3) Virgin layer

Energy conservation is equal to Equation (16).

Boundary conditions is equal to Equations (22) and (17).

For preheating stage, the Equations (16), (17) and (18) can be solved by iteration in each time step. The temperature distributions can be predicted quickly, the stability of the equations is reasonably good. The iteration computation can continue till the temperature reaches intumescence temperature. For intumescent combustion stage, two tasks have to do, i.e. to calculate the temperature distribution in the char and virgin layers and to track the intumescence interface. These two tasks were strongly coupled with each other by the boundary conditions. The method used here is to decouple the two tasks by iteration in each time step. The temperature field can be determined by char/virgin energy equations. The intumescence interface can be determined by Equation (23). Then mass loss rates can be calculated by Equation (24). In each iteration step of the decoupling process, the meshes of the sample need to be divided again because of the changeable sample thickness due to intumescence. Numerical results show that numerical calculation of the model is stable and sensitive.

\section{Experimental}

\subsection{Materials}

Materials used in the study include polypropylene (PP) supplied by Dalian Petrochemical Corporation; pentaerythritol (PER) supplied by Tianjin BoDi; ammonium polyphosphate (APP) supplied by Haida Chemical; zinc borate (ZB) supplied by Zhenjiang Sulfuric Acid Corporation; polyamide-6 (PA6) supplied by Shanghai Plastics Corporation; montmorillonite (MMT) supplied by Zhejiang Fenghong; zeolite (ZEO) prepared by laboratory.

MMT was used to prepare organic montmorillonite (OMMT) according to literature[18]. PA6 nano was prepared by extruding the mixture of PA6 and OMMT.

\subsection{Testing}

The FTT cone calorimeter was used for fire testing following the procedure defined in ISO-5660-1. 
The standard procedure used involves exposing specimens at heating flux levels of $50 \mathrm{~kW} \cdot \mathrm{m}^{-2}$, specimen sizes were $100 \mathrm{~mm} \times 100 \mathrm{~mm} \times 10 \mathrm{~mm}$. The experiments were conducted in air.

Differential scanning calorimetry NETZSCH DSC204 was used to measure heat capacity at a heating rate of $10 \mathrm{~K} / \mathrm{min}$ under air atmosphere. Heat capacity was computed using sapphire as reference.

The thermal conductivity of the sample is measured by using hot-wire method on a DRX-I thermal conductivity test instrument following the procedure defined in GB5990-86. The sample was measured from $293 \mathrm{~K}$ to $653 \mathrm{~K}$.

\section{Results and discussion}

Variation of heat capacity versus temperature of intumescent PP is shown in Figure 3, which shows that there are two peaks appeared during heating. When the temperature approaches $440 \mathrm{~K}$, PP begins to melt and heat is absorbed to complete phase change, resulting in a peak, which appears at about $440 \mathrm{~K}$. Then a rapid decrease of heat capacity is observed between $440 \mathrm{~K}$ and $460 \mathrm{~K}$. After that, heat capacity remains constant, but another peak appears at $500 \mathrm{~K}$ because of the pyrolysis of fire retardant. Figure 4 shows variation of heat capacity versus temperature of char. Heat capacity of char increases gradually when the temperature is below $400 \mathrm{~K}$, then decreases after that, indicating that the char is oxidized exothermally and results in the decrease of heat capacity of char. For simplicity, we take an approximate heat capacity value as the model's input parameter value, that is $C_{v}=$ $2600 \mathrm{~J} \cdot \mathrm{kg}^{-1} \cdot \mathrm{K}^{-1}, C_{c}=3000 \mathrm{~J} \cdot \mathrm{kg}^{-1} \cdot \mathrm{K}^{-1}$.

Variation of thermal conductivity $k$ versus temperature of intumescent PP as shown in Figure 5 shows that $k$ increases gradually with temperature when below about $373 \mathrm{~K}$. The value of the thermal con-

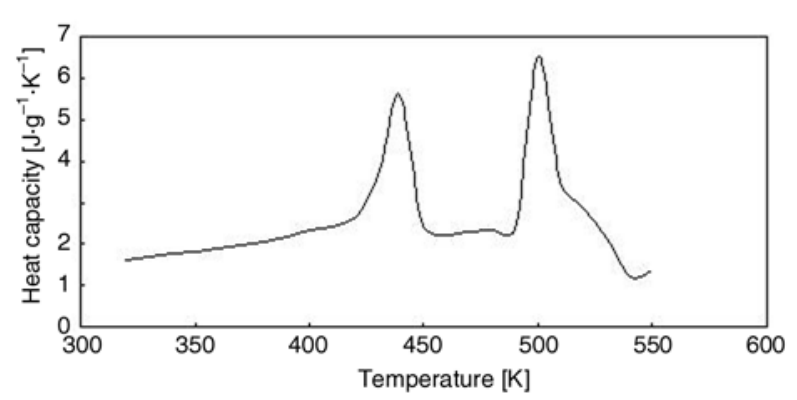

Figure 3. Variation of heat capacity versus temperature of intumescent PP

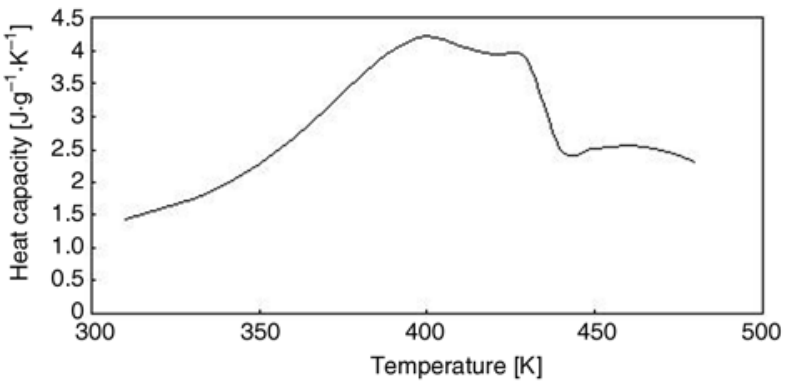

Figure 4. Variation of heat capacity versus temperature of char

ductivity initially increases until $373 \mathrm{~K}$ then decreases, goes through a minimum at about $553 \mathrm{~K}$ and after that increases up to about $653 \mathrm{~K}$. The sample begins to soften when the temperature above $373 \mathrm{~K}$, and melt at about $433 \mathrm{~K}$, which results in a decrease in thermal conductivity of the sample. When the temperature reaches $533 \mathrm{~K}$, PP begins to decompose and generates gases which form some bubbles remaining inside the sample and decrease the thermal conductivity. On the other hand when the temperature goes beyond $573 \mathrm{~K}$, char begins to form, and solid char improves the thermal conductivity of the sample. However, the thermal conductivity of char is still lower than that of the intumescent PP at low temperature. For simplicity, we take an approximate thermal conductivity value as the model's input parameter value, that is $k_{v}=0.5 \mathrm{~W} \cdot \mathrm{m}^{-1} \cdot \mathrm{K}^{-1}, k_{c}=0.2 \mathrm{~W} \cdot \mathrm{m}^{-1} \cdot \mathrm{K}^{-1}$.

The value of heat capacity and thermal conductivity based on the previous discussions are used in the model, all input parameters are listed as follows $[19,20]: k_{v}=0.5 \mathrm{~W} \cdot \mathrm{m}^{-1} \cdot \mathrm{K}^{-1} ; \rho_{v}=900 \mathrm{~kg} \cdot \mathrm{m}^{-3} ; c_{v}=$ $2600 \mathrm{~J} \cdot \mathrm{kg}^{-1} \cdot \mathrm{K}^{-1} ; k_{c}=0.2 \mathrm{~W} \cdot \mathrm{m}^{-1} \cdot \mathrm{K}^{-1} ; \rho_{c}=200 \mathrm{~kg} \cdot \mathrm{m}^{-3} ;$ $q_{p}=1 \cdot 10^{6} \mathrm{~J} \cdot \mathrm{kg}^{-1} ; h_{v}=h_{c}=10 \mathrm{~W} \cdot \mathrm{m}^{-2} \cdot \mathrm{K}^{-1} ; T_{p}=600 \mathrm{~K} ;$ $a_{v}=a_{c}=\varepsilon_{v}=\varepsilon_{c}=0.95 ; \quad T_{\infty}=298 \mathrm{~K} ; \quad c_{c}=$ $3000 \mathrm{~J} \cdot \mathrm{kg}^{-1} \cdot \mathrm{K}^{-1} ; \quad \Delta v_{f} / v_{0}=1.5 ; \quad$ Char yields $=$ $44.09 \% ; q=50 \mathrm{~kW} \cdot \mathrm{m}^{-2}$

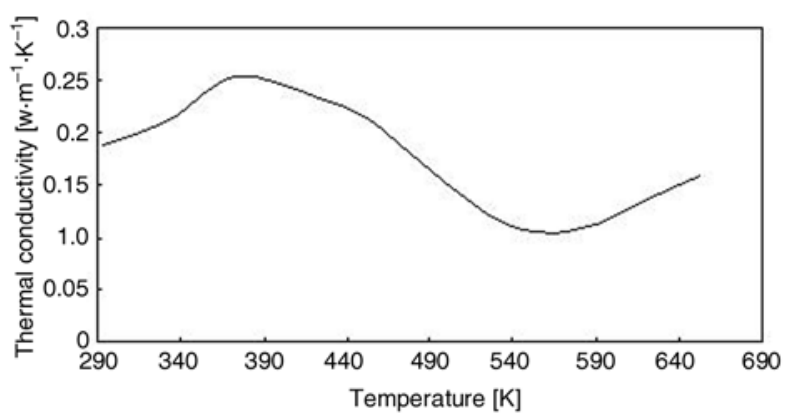

Figure 5. Variation of thermal conductivity versus temperature of the intumescent PP 
Predicted temperature curves at $1,8,9,10 \mathrm{~mm}$ locations from the bottom for polypropylene sample at incident heat flux of $50 \mathrm{~kW} \cdot \mathrm{m}^{-2}$ were shown in Figure 6. From the simulation curve, it is clear that when the temperature approaches intumescent temperature, the temperature increased very slowly due to the energy consumption for intumescent reaction. From the numerical prediction, it can be seen that the bending curve during the intumescent reaction was properly predicted by the model. Bending phenomenon is one of the characteristics of intumescent materials during fire. The present model can appropriately describe the intumescent behavior of polymer.

Meanwhile the thicknesses of the intumescent polypropylene during burning were measured for the validation of the model predictions. The validation results showed that the intumescent thicknesses predicted by the model were in fair good agreement with the experimental results as shown in Figure 7.

Figure 8 presents the comparison of the measured mass loss rates with the numerical mass loss rates obtained from the simulations. When the temperature of the sample reaches the pyrolysis temperature, the mass loss rate will increase quickly. The swelling of intumescent layer and the resulting multicellular char-forming limit both the heat transfer from the heat source to the substrate and the mass transfer from the substrate to the heat source, consequently protecting the underlying material. The mass loss rate will decrease after peak mass loss rate. Comparison with experimental results demonstrates that the predictions of mass loss rates

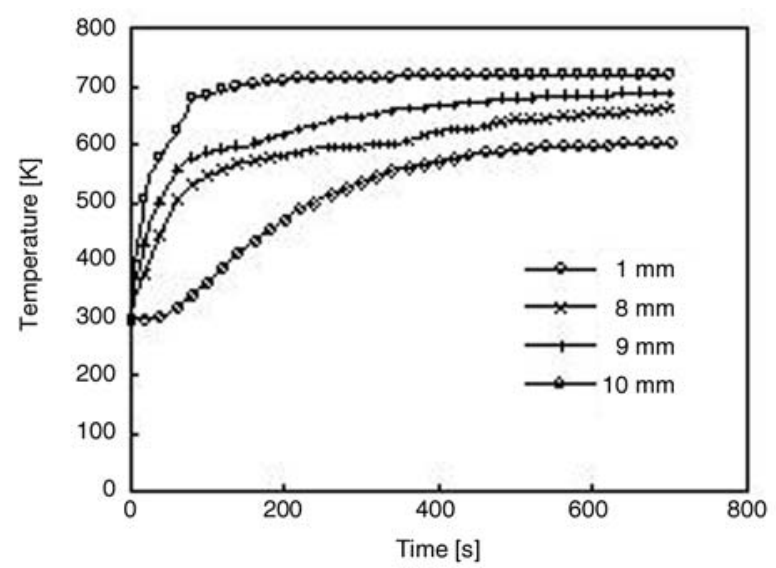

Figure 6. Predicted temperature curves at 1, 8, 9, $10 \mathrm{~mm}$ above the bottom of sample at incident heat flux of $50 \mathrm{~kW} \cdot \mathrm{m}^{-2}$

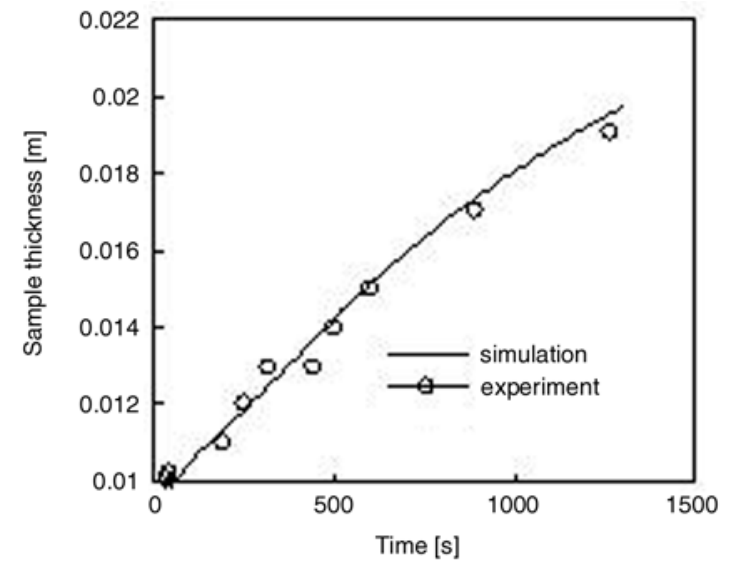

Figure 7. Comparison of measured and predicted materials thickness change with time for polypropylene at incident heat flux of $50 \mathrm{~kW} \cdot \mathrm{m}^{-2}$

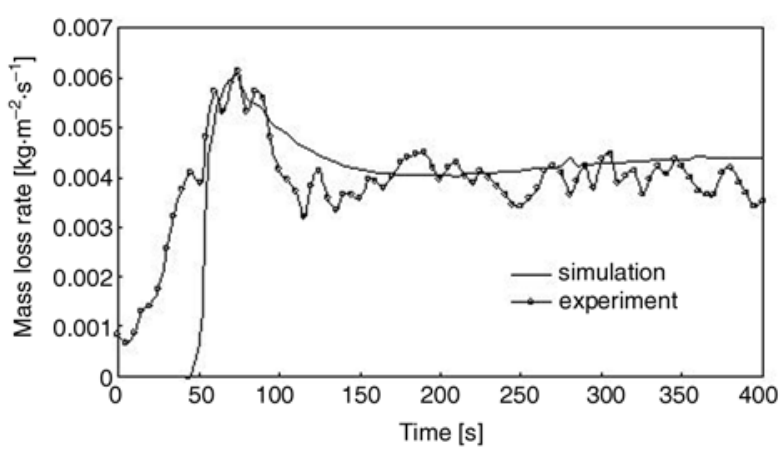

Figure 8. Comparison of the measured and predicted mass loss rates at incident heat flux of $50 \mathrm{~kW} \cdot \mathrm{m}^{-2}$

are in reasonably good agreement with the experiment.

\section{Conclusions}

A prediction model for the intumescence process in fire for intumescent flame retardant PP was developed. The model emphasizes the thermodynamic aspect of the intumescence process and a corresponding submodel is presented. Some key parameters such as thermal conductivity and heat capacity were measured and found these values would vary with time during experiment.

The validation results showed that the intumescent thicknesses and mass loss rates predicted by the model were in reasonably good agreement with the experimental results. The model was also used to predict the temperature distribution across the sample thickness during burning. The study shows that the present model can appropriately describe the intumescent behavior of polymer and numerically predict their intumescent thickness and temperature distribution in fire. 


\section{Acknowledgements}

The authors gratefully acknowledge the financial support of China NSFC Funds (No. 50176021) and China Special Doctorate Funds for Colleges and Universities.

\section{Nomenclatures}

a - absorbility

$c \quad-$ specific heat capacity $\left[\mathrm{J} \cdot \mathrm{kg}^{-1} \cdot \mathrm{K}^{-1}\right]$

$D \quad$ - the final depth of sample

$d \quad$ - the initial depth of sample

$h \quad$ - coefficient of convection heat $\left[\mathrm{W} \cdot \mathrm{m}^{-2} \cdot \mathrm{K}^{-1}\right]$

$k \quad-$ thermal conductivity $\left[\mathrm{W} \cdot \mathrm{m}^{-1} \cdot \mathrm{K}^{-1}\right]$

$L \quad$ - sample thickness [m]

$M \quad$ - molar weight $\left[\mathrm{g} \cdot \mathrm{mol}^{-1}\right]$

$m \quad-\operatorname{mass}[\mathrm{kg}]$

$\dot{m}^{\prime \prime} \quad$ - mass loss rate $\left[\mathrm{kg} \cdot \mathrm{m}^{-2} \cdot \mathrm{s}^{-1}\right]$

$n \quad$ - amount of gas [mol]

$P \quad$ - pressure of gas $[\mathrm{Pa}]$

$\dot{q}^{\prime \prime} \quad-$ external heat flux $\left[\mathrm{W} \cdot \mathrm{m}^{-2}\right]$

$q_{p} \quad-$ heat of pyrolysis $\left[\mathrm{J} \cdot \mathrm{kg}^{-1}\right]$

$q_{i} \quad$ - intumescent heat absorption $\left[\mathrm{J} \cdot \mathrm{kg}^{-1}\right]$

$Q \quad$ - heat absorption [kJ]

$R \quad$ - universal gas constant $\left[8.314 \mathrm{~kJ} \cdot \mathrm{kmol}^{-1} \cdot \mathrm{K}^{-1}\right]$

$s \quad$ - intumescent front [m]

$T_{P} \quad$ - intumescent temperature [K]

$t \quad$ - time [s]

$\Delta t \quad$ - time step [s]

$T \quad$ - temperature $[\mathrm{K}]$

$T_{\infty} \quad-$ ambient temperature [K]

$\begin{array}{ll}u & - \text { intumescent velocity }\left[\mathrm{m} \cdot \mathrm{s}^{-1}\right]\end{array}$

$U \quad$ - internal energy [kJ]

$v_{0} \quad$ - initial volume of sample $\left[\mathrm{m}^{3}\right]$

$v \quad-$ volume $\left[\mathrm{m}^{3}\right]$

$\Delta v_{f} / v_{0}-$ intumescent ratio

$W \quad-$ work $[\mathrm{kJ}]$

$x$ - coordinates axis [m]

$\Delta x \quad$ - distance step $[\mathrm{m}]$

$\varepsilon \quad-$ emissivity

$\rho \quad-$ density $\left[\mathrm{kg} \cdot \mathrm{m}^{-3}\right]$

$\sigma \quad-$ Stefan-Boltzmann constant $\left[5.669 \cdot 10^{-8} \mathrm{~W} \cdot \mathrm{m}^{-2} \cdot \mathrm{K}^{-4}\right]$

$\phi \quad-$ porosity

\section{Subscripts}

c - char material

$g \quad-$ gas

$s \quad-$ surface of sample

$v \quad$ - virgin material

$\infty \quad-$ ambient

\section{References}

[1] Jimenez M., Duquesne S., Bourbigot S.: Multiscale experimental approach for developing high-performance intumescent coatings. Industrial and Engineering Chemistry Research, 45, 4500-4508 (2006).

[2] Shuklin S. G., Kodolov V. I., Klimenko E. N.: Intumescent coatings and the processes that take place in them. Fibre Chemistry, 36, 200-205 (2004).

[3] Wei P., Li H., Jiang P., Yu H.: An investigation on the flammability of halogen-free fire retaudant PP-APPEG systems. Journal of Fire Sciences, 22, 367-377 (2004).

[4] Bourbigot S., Le Bras M., Dabrowski F., Gilman I. W., Kashiwagi T.: PA-6 clay nanocomposites hybrid as char forming agent in intumescent formulations. Fire and Materials, 24, 201-208 (2000).

[5] Shih Y. C., Cheung F. B., Koo J. H.: Theoretical modeling of intumescent fire-retardant materials. Journal of Fire Sciences, 16, 46-71 (1998).

[6] Le Bras M., Bourbigot S., Revel B.: Comprehensive study of the degradation of an intumescent EVAbased material during combustion. Journal of Materials Science, 34, 5777-5782 (1999).

[7] Di Blasi C.: Processes of flames spreading over the surface of charring fuels: effects of the solid thickness. Combustion and Flame, 97, 225-239 (1994).

[8] Parker W. J.: Predictions of the heat release rate of Douglas fire. in 'Proceedings of Fire Safety Science, Second International Symposium, Tokyo, Japan' 337-346 (1989).

[9] Anderson C. E., Wauters D. K.: A thermodynamic heat transfer model for intumescent systems. International Journal of Engineering Science, 22, 881-889 (1984).

[10] Buckmaster J., Anderson C., Nachman A.: A model for intumescent paints. International Journal of Engineering Science, 24, 263-276 (1986).

[11] Bourbigot S., Duquesne S., Leroy J-M.: Modeling of heat transfer of a polypropylene-based intumescent system during combustion. Journal of Fire Sciences, 17, 42-56 (1999).

[12] Staggs J. E. J.: A simplified mathematical model for the pyrolysis of polymers with inert additives. Fire Safety Journal, 32, 221-240 (1999).

[13] Di Blasi C.: Analysis of convection and secondary reaction effects within porous solid fuels undergoing pyrolysis. Combustion Science and Technology, 90, 315-340 (1993).

[14] Bhargava A., Griffin G. J.: A two dimensional model of heat transfer across a fire retardant epoxy coating subjected to an impinging flame. Journal of Fire Sciences, 17, 188-208 (1999).

[15] Bulter K. M., Baum H. R., Kashiwagi T.: Heat transfer in an intumescent material using a three-dimensional Lagrangian model. in 'International conference on fire research and engineering. Orlando, Florida USA', 261-266 (1995). 
[16] Zhang J., Ji K. J., Xia Y. Z.: Polymer combustion and fire-retardant technology. Chemical Industry Press, Beijing (2005).

[17] Wang Y., Zhang J., Lu X. D.: Simulated effects of melting behavior on combustion process of polymers. The Journal of Qingdao University of Science and Technology, 26, 312-316 (2005).

[18] Liu J. H., Zhang J., Li F.: Emulsion copolymerized styrene and butyl-crylate/organic-montmorillonite nanocomposites. China Synthetic Rubber Industry, 28, 90-93 (2005).
[19] Beyler C.: Thermal decomposition of polymers. in 'The SFPE handbook of fire protection engineering' (eds.: Di Nenno P. J., Beyler C. L., Custer R. L. P., Walton W. D., Watts J. M., Drysdale D. D., Hall J. R.), National Fire Protection Association, Quincy, 173-176 (1988).

[20] Sun S. C.: Applied Handbook of Plastics. Zhejiang Science and Technology Press, Hangzhou (1999). 